\title{
EL CRECIMIENTO ECONÓMICO ARGENTINO EN PERSPECTIVA HISTÓRICA
}

\author{
MARCELA A. GARCIA SEBASTIANI \\ I. U. Ortega y Gasset \\ ANTONIO SANTAMARÍA GARCÍA \\ Univ. Carlos III de Madrid e I. U. Ortega y Gasset
}

\section{INTRODUCCIÓN}

El estudio del crecimiento económico argentino ha despertado siempre el interés de la investigación. Sin embargo, carecía de una perspectiva de historia cuantitativa comparada que permitiese conocer su magnitud relativa. Con ese objetivo se celebró en la Universidad Carlos III de Madrid entre los días 26 y 27 de mayo de 1994 el seminario «El crecimiento económico argentino en perspectiva histórica», organizado por Leandro Prados de la Escosura y Carlos Newland, de aquella primera universidad, y por Roberto Cortés Conde, de la Universidad de San Andrés de la provincia de Buenos Aires.

Diferentes aspectos del crecimiento argentino fueron analizados en tres sesiones por varios investigadores. La reunión inicial, «La economia argentina en los siglos XIX y XX: una visión panorámica», estuvo a cargo de Roberto Cortés Conde y Daniel Diaz Fuentes. Zacarias Moutokias, María A. Irigoin y Carlos Newland fueron los ponentes de la segunda sesión: «La economía argentina antes y después de la independencia» ', y Gerardo della Paolera y Alan M. Taylor de la cuarta: «La Argentina en la economía internacional, 1860-1930». Aunque algunos trabajos incluyeron explícitamente una perspectiva compara$\mathrm{da}$-en concreto el de Diaz Fuentes con el caso español y el de Taylor con el australiano-, ésta fue aportada esencialmente por los comentaristas ${ }^{2}$. Final-

1 Esta sesión debia contar también con la participación de Samuel Amaral, quien finalmente no pudo asistir al seminario, pero envió su ponencia, lo que nos permite incluirla en la nota.

2 España (Leandro Prados de la Escosura y Blanca Sánchez Alonso), Brasil (Sergio Birchal),

Peru (Alfonso W. Quiroz) y Chile (Agustin Llona) fueron los paises objeto de la comparación. Además de los comentaristas mencionados, tres especialistas en historia económica de Espana: 
mente, una mesa redonda titulada «La economía argentina desde 1950» cerró el seminario intentando explicar las pautas del crecimiento hasta la actualidad y proponiendo algunas conclusiones. Entre ellas destacó especialmente la coincidencia de ponentes y comentaristas en que los factores de carácter institucional permiten entender gran parte de los problemas históricos del crecimiento económico argentino.

\section{LA ECONOMIA ARGENTINA EN LOS SIGLOS XIX Y XX: UNA VISIÓN PANORÁMICA}

La reconstrucción de una serie de PIB es fundamental para el estudio de las tendencias del crecimiento económico y las comparaciones internacionales. En el caso argentino, las primeras fuentes generales de referencia disponibles son los Censos de 1914 y 1935, y hasta hace poco tiempo careciamos de estimaciones para fechas anteriores a $1900^{3}$. El trabajo de R. Cortés Conde, «El crecimiento de la Argentina en el largo plazo", presenta una nueva serie para los años 1875-1985. Aporta información sobre el 60\% de los sectores económicos e incorpora datos sobre producto industrial, que no se pueden obtener directamente, pero es posible aproximarlos a través de las series de insumos ${ }^{4}$. No obstante y salvo algunas diferencias coyunturales, sus resultados son muy similares a los de las series elaboradas por la CEPAL y el BCRA para los años en que coinciden 5 .

Conocer la evolución del PIB desde 1875 permite una periodización del crecimiento económico argentino y, en el largo plazo, proporciona datos sobre algunos de los grandes problemas de debate historiográfico. En cuanto a la periodización, el análisis de Cortés Conde distingue dos grandes etapas: hasta 1929, la economía argentina creció el doble que la media mundial; después de 1929 la mitad (cuadro 1) 6.

Jordi Palafox, Pablo Martin Aceña y Gabriel Tortella moderaron la primera y tercera sesión y la mesa redonda con que se cerró el seminario, respectivamente. Otros tres especialistas en historia económica argentina completaron el panel de los participantes: Carlos D. Malamud como comentarista, y Carlos Rodriguez Braun y Ezequiel Gallo como ponentes de la mesa redonda. Gallo fue además el moderador de la segunda sesión.

3 Los trabajos de la CEPAL (1950, 1958 y 1978) reconstruyen series de producción para el periodo 1925.1948 y del PIB para 1900-1955 y 1900-1976, respectivamente. Un estudio del BCRA estima la renta nacional para los años 1935-1946.

4 La series de CEPAL carecen de datos sobre producto industrial.

${ }^{5}$ La serie está publicada en R. Cortés Conde y M. Harriague (1994).

6 En el análisis comparativo del crecimiento económico argentino, el autor consideró además los casos de los Estados Unidos, Francia, Reino Unido, Alemania, Australia e Italia. 


\section{CUADRO 1}

Tasa de crecimiento del PIB per cápita argentino, comparada con la de los países avanzados, 1875-1988 (en porcentajes)

\begin{tabular}{|c|c|c|c|}
\hline & Período & Argentina & $\begin{array}{c}\text { Paises } \\
\text { avanzados }\end{array}$ \\
\hline $1875-1988$ & & 1,0 & 1,5 \\
\hline $1875-1929$ & & 2,2 & 1,0 \\
\hline $1929-1988$ & & 0,9 & 2,0 \\
\hline
\end{tabular}

Fuente: Para Argentina, R. Cortés Conde y M. Harriague (1994). Para los países avanzados, A. Maddison (1991).

Si se estudian periodos más cortos (cuadro 2) 7 , la serie muestra un patrón de crecimiento tipo stop and go. Hubo crecimiento a largo plazo, pero siempre existieron dificultades para mantenerlo después de una década. Desde 1875 ha habido cuatro décadas buenas, tres de ellas en el siglo Xx, pero sólo una a partir de 1950. Hasta ese año el crecimiento económico argentino convergía con el del resto del mundo; desde entonces divergió y Argentina se convirtió en un país atrasado. La incapacidad de Argentina de sostener el motor del crecimiento por un periodo prolongado y la coincidencia de periodos de declinación económica con los mayores trastornos institucionales fueron, en definiti$\mathrm{va}$, las conclusiones generales de esta investigación.

\section{CUADRO 2}

Crecimiento del promedio del PIB argentino en distintos periodos

\begin{tabular}{|c|c|c|}
\hline & Período & $\begin{array}{c}\text { Crecimiento } \\
\text { promedio }\end{array}$ \\
\hline 1875.1896 & & 3,7 \\
\hline $1896-1912$ & & 2,3 \\
\hline $1912-1928$ & & $-0,1$ \\
\hline 1928.1948 & & 2,3 \\
\hline $1948-1961$ & & 0,3 \\
\hline $1961-1976$ & & 2,3 \\
\hline $1976-1987$ & & $-1,8$ \\
\hline
\end{tabular}

7 Los datos de los cuadros cuyas fuentes no se citan proceden de las ponencias que los autores presentaron en el seminario. 
En lo que respecta a los grandes temas de debate historiográfico, la nueva serie del PIB cuestiona la versión cepalina del crecimiento económico, pues confirma que el origen de la industrialización argentina fue anterior a la depresión de $1930^{8}$. Lo mismo puede decirse de la crisis del modelo primario-exportador con el que su economía se integró al mercado mundial durante la segunda mitad del siglo XIX 9 . Tanto estas conclusiones como la periodización, corroboran los resultados de los trabajos más recientes sobre la historia económica latinoamericana, aunque también reiteran algunas de sus carencias. La crítica a la visión tradicional sobre la crisis de 1930 no se corresponde con la propuesta de una nueva cronología para el estudio de la historia económica argentina en general y de la industrialización en particular. Sobre esta última también cabe señalar que avanzar en el conocimiento del tema requiere completar el análisis macroeconómico con más investigaciones a nivel sectorial y microeconómico ${ }^{10}$.

La ponencia de D. Díaz Fuentes revisa las «Estimaciones del crecimiento del PIB de Argentina, 1900-1950, un intento de comparación con España» ${ }^{11}$. Basada en un cuidadoso trabajo sobre la bibliografía y las fuentes, su investigación le permite presentar el primer cuadro sistematizado de la contaduría nacional (tabla input-output para 1950). Su análisis coincide con el de Cortés Conde al confirmar el atraso argentino desde 1950 (cuadro 3) 12; sin embargo profundiza con más detalle en las causas y, sobre todo, en el efecto de la crisis de 1930 y de las políticas económicas.

Evaluadas en perspectiva histórica y comparada, la crisis de 1930 y a las políticas económicas subsiguientes no son las únicas responsables de los problemas económicos actuales. El estudio de Díaz Fuentes los considera, asimismo, el resultado de un tipo de crecimiento con fluctuaciones (stop and go) des-

${ }^{8}$ Según Cortés Conde, la tasa de crecimiento anual del PIB por sector entre 1875 y 1929 fue:

\begin{tabular}{cccccc}
\hline Total & Industria & Agricultura & Ganaderia & Construcción & Comercio \\
\hline 6,3 & 7,0 & 11,2 & 3,0 & 7,7 & 6,1 \\
\hline
\end{tabular}

9 Esto coincide con los señalado pos distintos autores en la compilación de R. Thorp, comp. (1988) para la mayoria de los paises latinoamericanos.

10 Ver Ibidem.

11 Para las comparaciones internacionales utiliza los datos y la metodología de A. Maddison (1983) y R. Summers, I. Kravis y A. Heston (1980). La comparación con España la realiza fundamentalmente a través de las series reconstruidas por L. Prados de la Escosura (1993).

12 Tanto Cortés Conde como Diaz Fuentes confirman la opinión de A. Maddison (1991), quien señala dos periodos diferenciados en el crecimiento argentino: convergencia con el del resto de los paises avanzados hasta 1950 y divergencia a partir de esta última fecha. 
de los tiempos de la independencia ${ }^{13}$. En ese sentido, la crisis de 1930 es semejante a las de 1914 y 1820 y se confirma la opinión de C. Díaz Alejandro en cuanto a que las políticas económicas fueron eficaces contra la depresión de los años treinta (1913-1950 fue un período difícil para la economía mundial, pero Argentina se desenvolvió favorablemente). El problema es que aquéllas se perpetuaron posteriormente ${ }^{14}$.

\section{CUADRO 3}

Crecimiento PIB per cápita de distintos paises, 1870-1890/1973-1989

(medias anuales)

\begin{tabular}{rrrrrrrrrrr}
\hline Periodos & España & $\begin{array}{c}\text { Argen- } \\
\text { tina }\end{array}$ & Brasil & México & Corea & Japón & Italia & USA & RU \\
\hline $1870-1890$ & $\ldots$ & 0,5 & 1,9 & 0,2 & 0,4 & nd & 1,4 & 0,6 & 1,6 & 1,1 \\
$1890-1913$ & $\ldots$ & 2,2 & 2,0 & 0,4 & 4,7 & 1,8 & 0,4 & 1,9 & 1,0 & 0,9 \\
$1913-1950$ & $\ldots$ & 0,2 & 0,7 & 2,0 & 1,0 & $-0,2$ & 0,9 & 0,8 & 1,6 & 0,9 \\
$1950-1973$ & $\ldots$ & 5,1 & 2,1 & 3,8 & 3,1 & 5,2 & 8,0 & 5,0 & 2,2 & 2,5 \\
$1973-1989$ & $\ldots$. & 3,2 & $-2,2$ & 3,1 & 1,7 & 11,7 & 5,6 & 4,7 & 2,9 & 3,3 \\
\hline
\end{tabular}

FUENTE: A. Maddison (1993).

L. Prados de la Escosura y A. M. Taylor comentaron los trabajos de Cortés Conde y Díaz Fuentes. Ambos resaltaron la importancia de los factores institucionales para entender los problemas del crecimiento económico argentino. Taylor insistió en la tendencia «divergente» de dicho crecimiento comparado con el desarrollo mundial. Especialmente interesante fue la comparación de Prados de la Escosura con el caso español. En España, los momentos de menor crecimiento del PIB coinciden con fechas indicativas de la existencia de problemas institucionales. Estos problemas dificultaron la inversión, que a iguales tasas de ahorro fue menor, debido a que el aislamiento y el proteccionismo tradicional de la economía española y la utilización de los tipos de cambios como un arma política encarecieron el precio de los bienes de equipo ${ }^{15}$.

13 Ver D. Díaz Fuentes (1992).

14 C. Diaz Alejandro (1970).

15 Para las estimaciones del PIB español, ver L. Prados de la Escosura (1992). 


\section{LA ECONOMÍA ARGENTINA ANTES Y DESPUÉS DE LA INDEPENDENCIA}

La fuerte dependencia del crecimiento económico argentino de los factores de carácter institucional no se circunscribe sólo a los siglos XIX y XX. El trabajo de Z. Moutokias, «Crecimiento económico y politica imperial: Buenos Aires, 1760-1796», analiza uno de los temas más polémicos de la historia económica latinoamericana: las reformas borbónicas ${ }^{16}$. Su trabajo intenta demostrar que éstas no afectaron al crecimiento económico del Río de la Plata en las últimas décadas del siglo xvill. Por entonces, el sector exportador, especialmente el comercio del cuero (cuadro 4), tenía un papel fundamental en el desarrollo de la economía porteña. La importancia de ese producto resulta insólita, ya que se trata de una mercancía de mucho volumen y poco valor que, además, fue esencial para el crecimiento de Buenos Aires, pero no de la región rioplatense en su conjunto. Factores institucionales, en especial la importancia del puerto bonaerense en la economia virreinal de ese periodo, explican la paradoja. La creciente afluencia de metales preciosos del Alto Perú hacia dicho puerto - mercancías de poco volumen y mucho valor- permitió la exportación de productos locales y, con ello, el crecimiento del comercio, la población y los salarios y la ocupación de tierras. Por tanto, la aplicación del Reglamento de Libre Comercio no alteró sustancialmente el volumen y caracteristicas de la demanda externa. La expansión del comercio atlántico posterior a 1780 no fue tanto resultado del crecimiento de la economía rural del Río de la Plata como de la continuidad de una estructura comercial articulada en el siglo XviI.

La controversia del problema de la reformas borbónicas estuvo presente en la opinión de los comentaristas. C. D. Malamud señaló que la propia constatación de que hubo crecimiento indica que, al menos, aquéllas no lo perjudicaron. En la misma línea, comparándolo con el caso peruano, A. W. Quiroz advirtió que demostrar que las reformas no afectaron al crecimiento bonaerense requiere analizar sus efectos indirectos a través del comercio y el crédito ${ }^{17}$, y prolongar las series más allá de 1896 (límite cronológico del trabajo de Moutokias), para corroborar una hipótesis que, de confirmarse, cambiaría lo que sabe acerca de los costes económicos de la independencia ${ }^{18}$.

16 Sobre la discusion historiográfica acerca del efecto de las reformas borbónicas en América Latina, ver P. Pérez Herrero (1992).

17 Sobre el caso peruano, ver A. W. Quiroz (1993).

18 Sobre esta tema, ver Prados de la Escosura y Amaral (1993). 


\section{CUADRO 4}

Exportación de cueros desde Buenos Aires, 1756/59-1795/76

(valor en miles de pesos de 8 reales)

\begin{tabular}{|c|c|c|}
\hline & Período & $\begin{array}{c}\text { Exportaciones } \\
\text { decueros }\end{array}$ \\
\hline $1756-1759$ & & 213 \\
\hline $1760-1764$ & 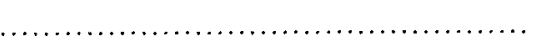 & 110 \\
\hline $1765-1769$ & & 140 \\
\hline $1770-1774$ & ...... & 163 \\
\hline $1775-1779$ & & 178 \\
\hline $1780-1784$ & & 235 \\
\hline $1785-1789$ & $\ldots \ldots \ldots$ & 265 \\
\hline $1790-1794$ & $\ldots \ldots \ldots \ldots \ldots \ldots$ & 401 \\
\hline $1795-1796$ & $\ldots \ldots \ldots \ldots \ldots \ldots \ldots, \ldots \ldots \ldots \ldots \ldots \ldots \ldots$ & 550 \\
\hline
\end{tabular}

El resto de los ponentes de la segunda sesión analizaron problemas posteriores a la independencia. M. A. Irigoin, «The Economic Consecuencies of State Financing, 1820-1860», propone estudiar el financiamiento del proceso de construcción del Estado vinculado al análisis del endeudamiento interno y de las políticas monetarias de los gobiernos ${ }^{19}$. De su análisis se desprende que el estancamiento económico puede ser consecuencia del deterioro de la base contractual de la economía. Un Estado débil como el argentino es un estado con fuerte propensión inflacionaria. Este ejercicio, aunque incompleto ${ }^{20}$, permite algunas hipótesis válidas para el estudio de los problemas actuales, que vuelven a situar los factores institucionales como la razón principal del atraso económico argentino.

Las contribuciones más importantes del seminario para el conocimiento de la historia económica argentina del siglo xix fueron los trabajos de C. Newland y S. Amaral que, además, se complementan. Hay buenos estudios sobre el periodo anterior a $1870^{21}$, pero carecemos de una perspectiva institucional y na-

19 La autora considera las valiosas aportaciones sobre las consecuencias económicas de la independencia y de la construcción del Estado nacional en Argentina de J. Alvarez (1914), M. Burgin (1975), T. Halperin (1982) y S. Amaral (1993).

${ }^{20}$ La hipótesis es interesante, pero de momento las carencias del trabajo impiden demostrarla: adolece de información cuantitativa suficiente y de un modelo de análisis económico. En su comentario, C. D. Malamud señaló, incluso, la necesidad de ampliar cronológicamente el estudio que, a pesar de lo que anuncia el titulo de la ponencia, se limita a la década de 1850 .

21 Ver, por ejemplo, M. Burgin (1975) y T. Halperin (1980). 
cional que tome en consideración el cambio estructural a largo plazo desde la independencia. Newland se plantea ese problema e intenta una aproximación a través de la relación «Población y crecimiento económico: Argentina, 18201870». Los comentarios sobre el trabajo señalaron que la debilidad de la base empírica no resta valor a un esfuerzo, que además no difiere mucho del realizado para otros paises. Los datos disponibles, trabajados a nivel regional (litoral e interior), permiten distinguir dos grandes etapas. Entre 1820-1850 se mantuvo estancado el producto per cápita y probablemente también los salarios reales, debido a que las guerras de independencia y civiles, los bloqueos económicos externos y el proteccionismo redujeron el efecto positivo de la liberalización del comercio exterior tras la emancipación. Entre 1850-1870, se aceleró el crecimiento de las exportaciones y de los salarios reales, disminuyó la protección y se eliminaron las trabas para el comercio interno, mejorando los términos de intercambio (cuadro 5). El crecimiento vegetativo de la población y la inmigración acompañó al de los demás indicadores en las dos etapas y fue paralelo a la incorporación de mejoras tecnológicas, al avance de la roturación y de la urbanización, especialmente de Buenos Aires y el litoral. Finalmente, las diferencias tradicionales entre el litoral (moderno) y el interior (atrasado) se acentuaron también con el tiempo.

\section{CUADRO 5}

Tasa de crecimiento del comercio argentino y términos de intercambio con Gran Bretaña (1920-1870)

\begin{tabular}{cccccc}
\hline Periodo & $\begin{array}{c}X \\
\text { totales }\end{array}$ & $\begin{array}{c}X \\
\text { per cápita }\end{array}$ & $\begin{array}{c}\text { Comercio } \\
\text { mundial }\end{array}$ & $\begin{array}{c}\text { Relación real } \\
\text { de intercambio }\end{array}$ \\
\hline $1820-1850$ & $\ldots \ldots \ldots \ldots \ldots \ldots \ldots \ldots \ldots \ldots$ & 2,5 & 0,1 & 4,0 & 100,0 \\
$1850-1870$ & $\ldots \ldots \ldots \ldots \ldots \ldots \ldots \ldots \ldots$ & 8,3 & 5,2 & 4,4 & 109,3 \\
$1820-1870$ & $\ldots \ldots \ldots \ldots \ldots \ldots \ldots \ldots \ldots$ & 4,8 & 4,2 & 4,2 & 139,3 \\
\hline
\end{tabular}

El análisis de la población y del comercio exterior permite cuatro interesantes conclusiones. En primer lugar, parece que Argentina creció al mismo ritmo que la media mundial entre 1820-1870, aunque, a la inversa de lo que señalaba Cortés Conde para 1875-1988, con dos períodos bien diferenciados que se compensan: entre $1820-1850$ creció la mitad que la media mundial y entre 1850-1870 el doble. En segundo lugar, esto indica que el crecimiento 
económico comenzó antes de lo que ha señalado la historiografia. En tercer lugar, la estructura económica argentina era muy similar en 1870 y 1820 . Por último, las causas del atraso parecen encontrarse en el periodo posterior a la independencia y son eminentemente institucionales ${ }^{22}$.

El trabajo de S. Amaral, «Trade and Markets: the Performance of Buenos Aires Livestock Byproducts, 1810-1870», analiza el comercio bonaerense. La implantación del libre comercio tras la independencia inauguró un periodo de crecimiento de las exportaciones gracias a que los costos de producción fueron suficientemente bajos para compensar los fletes y demás gastos de comercialización. En términos generales, Gran Bretaña y los EE.UU. se mostraron inmediatamente como principales mercados para la producción porteña (cuadro 6). No obstante, la estructura geográfica del comercio varió dependiendo del tipo de producto y del tiempo. Esto permite afirmar que no hubo un patrón común en la evolución de los mercados. Algunos, como el del sebo y el de la lana en Gran Bretaña, estaban desarrollados antes de iniciarse las exportaciones de Buenos Aires. En el primer caso fue posible capturarlo, en el segundo no. Otros, como el de la lana en Bélgica, aumentaron su demanda al mismo ritmo con el que se elevaron las exportaciones porteñas.

\section{CUADRO 6}

Exportaciones de cuero, lana y sebo destinadas a Gran Bretaña y los Estados Unidos, 1822-1862 (en porcentajes del valor total)

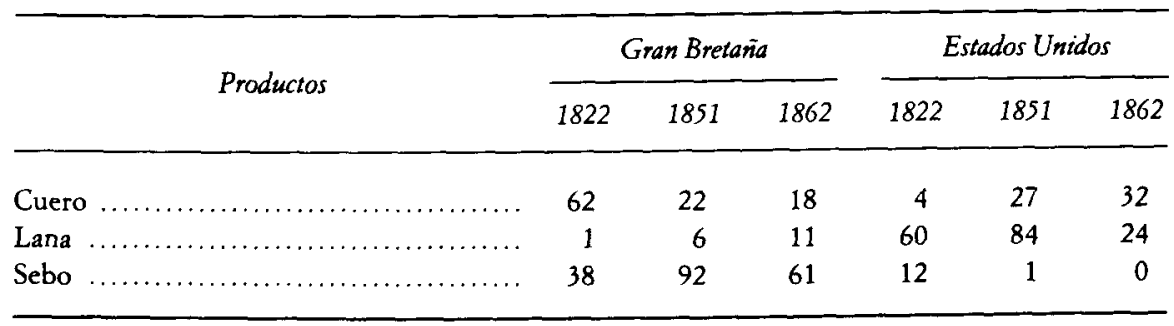

Hubo también casos en los que un producto de primera importancia se estancó o redujo su participación en uno de los mercados principales en un momento dado, pero sus exportaciones totales aumentaron. Esto permite señalar que la especialización de la producción bonaerense se mostró muy flexible

22 Esto es muy similar a lo que señala L. Prados de la Escosura (1988) para el caso español. 
entre 1820 y 1870 . Los principales importadores eran los EE.UU., Gran Bretaña, Francia y Bélgica (cuadro 7); sin embargo, ninguno de ellos fue el destino primordial de todos los productos al mismo tiempo. Esta estrategia, además, no resultó de una planificación consciente, sino del óptimo aprovechamiento de las ventajas comparativas, comportamiento que concuerda con la tesis de Hirschman sobre la diversificación de los mercados de exportación como factor esencial para crecimiento económico de la periferia ${ }^{23}$.

\section{CUADRO 7}

Estructura geográfica del comercio de Buenos Aires, 1822-1872

(expresa en porcentajes del valor de las exportaciones totales)

\begin{tabular}{|c|c|c|c|c|}
\hline Países & 1822 & 1842 & 1851 & 1872 \\
\hline Gran Bretaña & 47 & 24 & 27 & 17 \\
\hline Francia $\ldots \ldots \ldots$ & 3 & 26 & 10 & 23 \\
\hline Estados Unidos . & 9 & 20 & 30 & 10 \\
\hline Bélgica $\ldots \ldots \ldots \ldots \ldots \ldots \ldots \ldots \ldots$ & 3 & 3 & 12 & 36 \\
\hline
\end{tabular}

\section{ARGENTINA EN LA ECONOMÍA INTERNACIONAL, 1860-1930}

Estudiar la política monetaria es importante siempre y cuando ésta no actúe de forma neutral y desestabilice el crecimiento económico. En opinión de G. della Paolera, «Monetary and Banking Experiments in Argentina: 1861$1930 »$, ese fue el caso argentino ${ }^{24}$. Un pais relativamente pequeño, primario exportador, alejado del centro económico mundial, que no formaba parte de un imperio ni producia metales preciosos, diseñó unilateralmente su política partiendo de que su base monetaria dependía de la balanza de pagos y de que un régimen fiscal y monetario creible era esencial para la integración de su economía en el mercado mundial. Un modelo econométrico de equilibrio general sobre el supuesto de que el objetivo del gobierno y de los agentes económicos fue maximizar la relación dinero, deuda e impuestos permite explicar la política monetaria y distinguir y analizar detalladamente cuatro grandes etapas.

23 A. O. Hirschman (1958).

24 G. della Paolera (1988) realizó su tesis doctoral sobre este problema. 
Argentina estuvo dentro del patrón oro hasta el crac Baring (1890-91) ${ }^{25}$. El colapso financiero - tema central del estudio- tuvo su origen en el diseño de una inconsistente política fiscal y monetaria interna. Para comprender sus causas es necesario preguntarnos sobre las razones por las que los agentes económicos dejaron de tener expectativas racionales y pasaron a tener expectativas inflacionarias. La respuesta es que el gobierno agotó otras posibilidades para hacer frente a los problemas económicos y sólo le quedó el recurso del impuesto inflacionario.

En el cuadro 8 anotamos la relación entre el nivel de precios y la tasa de cambio entre 1884 y 1910 . El análisis de los otros tres periodos es más descriptivo que el de la etapa 1861-1890. Aunque Argentina no retornó al patrón oro hasta 1899, en los años noventa diseñó una política monetaria ortodoxa: deflación y ajuste económico. La vuelta al oro se realizó, además, dentro de un marco restrictivo, limitada por el comportamiento de la balanza de pagos. Cuando el resultado de ésta era negativo, no se garantizaba la convertibilidad del papel moneda. Finalmente, Argentina sufrió más que otros paises latinoamericanos los efectos de la Primera Guerra Mundial y de la crisis del sistema financiero británico en 1920 y fue el primer pais de la región en abandonar el patrón oro durante la depresión de los años treinta, aunque también era el mayor deudor ${ }^{26}$.

Los comentarios sobre la ponencia de Della Paolera señalaron la necesidad de un análisis más detenido de las variables políticas. Agustín Llona comparó el caso argentino con el chileno, resaltando la semejanza en la relación déficit público, política bancaria y sector externo en ambas economías.

El trabajo de A. M. Taylor, «Mass Migration to Distant Souther Shores: Argentina and Australia, 1870-1939», cerró la tercera sesión del seminario. Salvo para los EE.UU. y Australia, carecemos de estudios cuantitativos sobre las causas de la inmigración que permitan comparaciones internacionales. Taylor elabora un modelo econométrico y compara los casos de Argentina y Australia ${ }^{27}$. La razón de este análisis es la importancia de la inmigración en el crecimiento económico argentino en las últimas décadas del siglo XIX, además de ser el principal componente de la población en el período 1880-1913 (cuadro 9).

25 Sobre la crisis financiera de 1890-1891 en Argentina, ver A. Ford (1962) y J. Williams (1969). Para América Latina en general, C. Marichal (1988).

${ }^{26}$ La coincidencia de estas conclusiones con las del trabajo de Cortés Conde, quien señalaba que muchos de los efectos que tradicionalmente se achacaban a la crisis de 1930 estaban presentes desde la Primera Guerra Mundial, confirma la valoración de Della Paolera acerca de la importancia del análisis monetario en aquellos casos en que su comportamiento no es neutral para el crecimiento económico.

${ }^{27}$ Para el caso australiano, ver A. M. Taylor (1991). 


\section{CUADRO 8}

Nivel de precios y tasa de cambio en Argentina en años significativos, 1884-1910 $(1884=1)$

\begin{tabular}{|c|c|c|c|}
\hline & Años & $\begin{array}{c}\text { Nivel de } \\
\text { precios }\end{array}$ & $\begin{array}{l}\text { Tasa de } \\
\text { cambio }\end{array}$ \\
\hline 1884 & & 1,0 & 1,0 \\
\hline 1885 & & 1,3 & 1,2 \\
\hline 1888 & $\cdots \cdots$ & 1,5 & 1,2 \\
\hline 1891 & $\ldots \ldots$ & 3,8 & 3,2 \\
\hline 1893 & $\ldots \ldots \ldots \ldots \ldots \ldots \ldots \ldots \ldots \ldots \ldots \ldots \ldots$ & 3,7 & 2,4 \\
\hline 1896 & & 3,0 & 2,4 \\
\hline 1899 & & 2,3 & 2,0 \\
\hline 1904 & $\ldots \ldots \ldots \ldots \ldots \ldots \ldots \ldots \ldots \ldots \ldots \ldots$ & 2,3 & 2,3 \\
\hline 1906 & $\ldots \ldots \ldots \ldots \ldots \ldots \ldots \ldots \ldots \ldots \ldots \ldots \ldots$ & 2,5 & 2,3 \\
\hline 1910 & $\ldots \ldots \ldots \ldots \ldots \ldots \ldots \ldots \ldots \ldots \ldots \ldots \ldots$ & 2,9 & 2,3 \\
\hline
\end{tabular}

\section{CUADRO 9}

Tasas de inmigración neta, crecimiento de la población y participación de la primera en la segunda para varios países entre 1890 y 1913 (en porcentajes)

\begin{tabular}{|c|c|c|c|}
\hline Paises & $\begin{array}{l}\text { Tasa de } \\
\text { inmigración neta } \\
\text { (1) }\end{array}$ & $\begin{array}{l}\text { Tasa de crecimiento } \\
\text { de la población } \\
\text { (2) }\end{array}$ & $\begin{array}{l}\text { Participación } \\
\text { (1) en (2) }\end{array}$ \\
\hline Argentiña & 1,32 & 1,81 & 73 \\
\hline Australia $\ldots \ldots \ldots \ldots \ldots \ldots \ldots \ldots \ldots \ldots \ldots \ldots \ldots \ldots \ldots$ & 0,31 & 0,96 & 33 \\
\hline 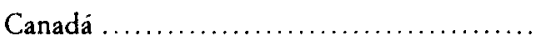 & 1,49 & 1,06 & 141 \\
\hline Estados Unidos $\ldots \ldots \ldots \ldots \ldots \ldots \ldots \ldots \ldots \ldots \ldots \ldots \ldots$ & 0,41 & 0,98 & 42 \\
\hline
\end{tabular}

FUENTE: J. Williamson (1991).

Según Taylor, no se puede hablar de un mercado internacional de trabajo en las últimas décadas del siglo XIX. La inmigración, pues, tuvo múltiples causas, entre las que se encuentran factores raciales, nacionales y culturales. Un análisis estadístico de estas variables, junto con el diferencial salarial (cuadro 10) explica por qué los inmigrantes australianos procedian de Gran Bretaña, mientras los argentinos lo hacían de España e Italia. Frente a lo que se ha dicho en algunas ocasiones, el flujo de la emigración a Argentina no fue comple- 
tamente elástico. Por tanto, los emigrantes de la Europa suroccidental podrían haberse desplazado a Australia, donde, además, el diferencial salarial era mayor. Si no lo hicieron - según Taylor- fue porque el coste del viaje fue relativamente más caro para estos últimos, más pobres que los británi$\cos$, y porque se vieron desfavorecidos por las leyes de inmigración australianas.

\section{CUADRO 10}

Diferencial salarial entre Australia y Gran Bretaña y entre Argentina, Italia y España, 1890-1940 (en porcentajes)

\begin{tabular}{lrrrrrrr}
\hline Años & 1890 & 1900 & 1910 & 1920 & 1930 & 1940 \\
\hline Australia-Gran Bretaña $\ldots \ldots \ldots \ldots \ldots \ldots \ldots$ & 49 & 30 & 30 & 7 & 30 & 30 \\
Argentina-Italia $\ldots \ldots \ldots \ldots \ldots \ldots \ldots \ldots \ldots \ldots$ & 140 & 220 & 110 & 30 & 120 & 140 \\
Argentina-España $\ldots \ldots \ldots \ldots \ldots \ldots \ldots \ldots \ldots \ldots$ & 70 & 170 & 110 & 60 & 110 & nd \\
\hline
\end{tabular}

Los comentaristas valoraron el esfuerzo de cuantificación realizado por Taylor. Su ponencia fue la más cliométrica de la reunión y también la más discutida. B. Sánchez Alonso, en el mejor trabajo de comentarista del seminario, tildó de tópicas sus afirmaciones sobre las diferencias entre los emigrantes británicos y los españoles e italianos. En primer lugar, duda que estos últimos fuesen más pobres. El mismo hecho de que emigrasen demuestra que las diferencias no debieron ser muy grandes. En segundo lugar, sus propios estudios para el caso español prueban que no fueron los más pobres los que optaron por abandonar el país. Además, habría que considerar la cuestión de las redes de inmigrantes. Taylor la omite, pero es esencial para explicar el destino de la población y, en algunos casos, la financiación de los gastos del viaje ${ }^{28}$. En tercer lugar, estudiando ex post la dispersión de la emigración, los que más oportunidades tuvieron, sin duda, no fueron los británicos, sino los italianos. Finalmente, en lo que respecta al argumento sobre las leyes de inmigración australianas, Sanchez Alonso opina que éstas no perjudicaron a los españoles y transalpinos, puesto que su objetivo era fundamentalmente impedir la entrada de emigrantes del Pacifico Sur.

28 Ver B. Sánchez Alonso (1988). 


\section{LA ECONOMÍA ARGENTINA DESDE 1950}

La última sesión del seminario se dedicó a analizar la economía argentina desde 1950 a la luz del estudio del crecimiento en perspectiva histórica comparada. Moderada por G. Tortella, contó con la participación de R. Cortés Conde, G. della Paolera, C. Rodriguez Braun y E. Gallo. Aunque la discusión fue abierta, tomó como referencia un trabajo de Cortés Conde ${ }^{29}$. El autor senala que la severa crisis fiscal de los años ochenta tuvo caracteres imprecedentes e influencias muy negativas en Argentina: alto grado de inconstancia en la aplicación de las políticas económicas, destrucción del marco institucional y desaliento de la inversión y del crecimiento económico. En conclusión, y coincidiendo con lo señalado por la mayoria de los ponentes, la formulación de politicas y la creación de instituciones no son posibles sin la regulación de un marco institucional estable en el que las prácticas arbitrarias no creen incertidumbre. Para ello resulta imprescindible un consenso en el sentido del reciente premio Nobel D. C. North, que aproxime los costos y los beneficios privados ${ }^{30}$.

Rodriguez Braun retomó las conclusiones de Diaz Fuentes proponiendo un peculiar teorema interpretativo. Los problemas del crecimiento económico argentino - dijo- se explican por la peculiar combinación de dos factores: el miedo y la moda. El miedo lo provocó la crisis de 1930. Cuando llegó Perón, la reacción contra el mismo (léase las políticas económicas de cuya perpetuación hablaba Díaz Alejandro) ${ }^{31}$, se había convertido en moda ${ }^{32}$. C. D. Malamud cuestionó esta interpretación, señalando no haber encontrado en la prensa argentina de la época referencia alguna al citado miedo. Sin embargo, Rodríguez Braun contestó que la depresión de 1930 provocó una desconfianza sin precedentes en las instituciones que hasta ese momento habian regido el mercado. Tortella y Della Paolera ahondaron en esta opinión, señalando la existencia de una ideología victimista y de una aversión al riesgo de la sociedad argentina. Della Paolera, en el comentario más interesante de la mesa redonda, reflexionó sobre el valor económico de la incertidumbre y sobre la dificultad de hacer coincidir la racionalidad política con la económica. En muchos casos, las soluciones que Argentina ha ofrecido para los problemas económicos han sido eminentemente políticas. De esta lección del pasado es posible deducir que las

29 R. Cortés Conde (1992).

30 D. C. North (1990).

31 C. Diaz Alejandro (1970).

32 Este argumento coincide también con la reciente tesis de $\mathrm{P}$. H. Lewis, que presenta a Perón como un oportunista, P. H. Lewis (1990). 
actuales propuestas de ajuste y de reforma dependen de su rapidez, constancia y envergadura. Para que ambas racionalidades coincidan es preciso eliminar incertidumbre y conseguir que, aunque todos pierdan en términos absolutos, también obtengan beneficios marginales.

La coincidencia de los ponentes en los factores institucionales como causas de los problemas del crecimiento económico argentino fue probablemente la conclusión más interesante del seminario. Ezequiel Gallo insistió en la idea del consenso político y en el problema de la múltiple racionalidad. La inestabilidad del mercado institucional - dijo - ha impedido el crecimiento económico, y viceversa. La solución definitiva de las dificultades por las que atraviesa Argentina depende de que se puedan hacer compatibles ambas reformas.

\section{BIBLIOGRAFÍA}

Álvarez, Julián (1914): Estudio sobre las guerras civiles argentinas, Buenos Aires, Ed. Roldán.

Amaral, Samuel (1993): «El mercantilismo y la libertad: consecuencias económicas de la independencia argentina», en Prados de la Escosura, L., y S. Amaral, eds. (1993).

BCRA (Banco Central de la República Argentina): La renta nacional de la República Argentina, 1935-1945, Buenos Aires.

BuRgin, Miron (1975): Aspectos económicos del Federalismo argentino, Buenos Aires, Ed. Hachette.

CEPAL (Comisión Económica para América Latina) (1950): Estudio económico de América Latina, Nueva York.

- (1958): El desarrollo económico de la Argentina, Santiago de Chile.

- (1978): Serie bistórica de crecimiento del producto de América Latina, Cuadernos de la CEPAL, núm. 3, Santiago de Chile.

Cortés CONDE, Roberto (1992): «Growth and Stagnacion in Argentina», en Teitel, S., ed. (1992).

- y Harriague, Marcela (1994): Estimaciones del PIB de Argentina, 1875-1935 (papeles de trabajo, Univ. de San Andrés).

Della Paolera, Gerardo (1988): How the Argentine Economy Performed During the International Gold Standard: A Reexamination (tesis doctoral inédita, Univ. of Chicago).

Diaz Alejandro, Carlos (1970): Essay on the Economic History of the Argentina Republic, New Haven, Yale Univ. Press.

DIAZ FuENTES, Daniel (1992): Cambios estructurales en América Latina: un análisis comparado de la evalución económica y fiscal de Argentina, Brasil y México en el periodo de entreguerras (tesis doctoral inédita, Univ. de Alcalá de Henares).

ForD, Alec (1962): The Gold Standard, 1880-1914: Britain and Argentina, Oxford, Clarendon Press.

Hal.PERIN, Tulio (1980): De la Revolución de la Independencia a la Confederación Rosista, Buenos Aires. 
- (1982): Guerra y finanzas en la organización del estado argentino, Buenos Aires, Ed. Belgrano.

Hirschman, Albert O. (1958): The Strategy of Economic Development, Londres.

Lewis, Paul H. (1990): The Argentine's Crisis Capitalism, Chapell Hill, Univ. of North Caroline Press.

MADDIson, Angus (1983): «A Comparission of the Level of GDP Per Capita in Developed and Developing Countries, 1700-1980m, The Journal of Economic History, V. 43, núm. 1.

- (1991): Dynamic Forces. Capitalism Development, Nueva York, Oxford Univ. Press.

- (1993): «Long Economic Growth in the European Periphery», European Historical Economics Society Workshop, La Coruña, Univ. Internacional Menéndez Pelayo.

Marichal, Carlos (1988): Historia de la deuda externa en América Latina, Madrid, Alianza América.

NoRTH, Douglass C. (1990): Institutions, Institutional Change, and Economic Performance, Cambridge Univ. Press.

Pérez Herrero, Pedro (1992): Comercio y mercados en América Latina colonial, Madrid, Ed. Mapfre.

Prados de la Escosura, Leandro (1988): De imperio a nación, Madrid, Alianza Ed.

- (1992): Spain's Real Gross Domestic Product, 1850-1990: a New Index, Madrid (mimeo).

— y AmARAl, Samuel, eds. (1993): La independencia americana: consecuencias económicas, Madrid, Alianza.

Quiroz, Alfonso W. (1993): «Consecuencias económicas y financieras del proceso de independencia en Perú», en Prados de la Escosura, L., y Amaral, S., eds. (1993).

SAnCHEZ AlONSO, Blanca (1988): «La emigración española a la Argentina, 1880-1930», en SANChEZ-Albornoz, N., ed. (1988).

Summers, R; Kravis, I., y Heston, A. (1880): «International Comparission of Real Product and Its Composition: 1950-1977», Review of Income and Wealth, V. 26, núm. 1.

SÁnCHEZ-Albornoz, Nicolás, ed. (1988): España bacia América: la emigración en masa, 1880-1930, Madrid, Alianza Ed.

TEITEL, Simon, ed. (1992): Towards a new development strategy for Latin America (Pathways from Hirschman's Thought, Washington, Inter-American Development Bank and Johns Hopkins Univ. Press.

TAYLOR, Alan M. (1991): Patterns of Trade Migration to Australia, 1870-1914 (trabajo inédito, Harvard Univ. Press).

THORP, Rosemery, ed. (1988): América Latina en los años treinta, México, FCE.

Williams, John (1969): Argentine Trade under inconvertible Paper Money, Nueva York, Greenwood Press.

Williamson, Jeffrey (1991): The Evolution of Global Labor Markets in the First and Second World Since 1830: Background Evidence and Hypotbeses, Harvard Inst. of Economic Research, Discussion Paper Series, núm. 1571, Harvard Univ. 